\title{
Boosting Deuteron Polarization in HD Targets: Experience of moving spins between $H$ and $D$ with RF methods during the E06-101 experiment at Jefferson Lab
}

\author{
X. Wei ${ }^{* a}$, C.D. Bass ${ }^{a \dagger}$, A. D'Angelo ${ }^{b}$, A. Deur ${ }^{\mathrm{a}}$, G. Dezern ${ }^{\mathrm{a}}$, T. Kageya ${ }^{\mathrm{a}}$, V. Laine ${ }^{\mathrm{a}, \mathrm{c}}$, \\ M.M. Lowry ${ }^{\mathrm{a}}$, A.M. Sandorfi ${ }^{\mathrm{a}}$, R.W. Teachey ${ }^{\mathrm{a}}$, H. Wang ${ }^{\mathrm{a}}$ and C.S. Whisnant ${ }^{\mathrm{d}}$

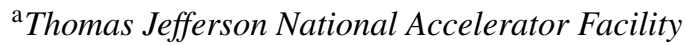 \\ 12000 Jefferson Ave., Newport News, VA 23606, USA \\ ' Universita' di Roma2 "Tor Vergata" and INFN Sezione di Roma, Roma, Italy \\ ${ }^{\mathrm{c}}$ Universite Blaise Pascal, Clermont-Ferrand, France \\ ${ }^{\mathrm{d}}$ Department of Physics, James Madison University, Harrisonburg, VA 22807, USA \\ E-mail: xwei@jlab.org
}

\begin{abstract}
Solid HDice targets are polarized by bringing the HD crystal to thermal equilibrium at low temperature and high magnetic field, typically 10-20 mK and 15 Tesla, at Jefferson Lab. In this regime, due to its smaller magnetic moment, the resulting polarization for $\mathrm{D}$ is always at least three times smaller than for $\mathrm{H}$. The controlled amount of polarizing catalysts, $\mathrm{o}-\mathrm{H}_{2}$ and $\mathrm{p}-\mathrm{D}_{2}$, used in the process of reaching a frozen-spin state, further limit the maximum achievable D polarization. Nonetheless, $\mathrm{H}$ and $\mathrm{D}$ polarizations can be transferred from one to the other by connecting the $\mathrm{H}$ and D sub-states of the HD system with RF. In a large target, the RF power needed for such transitions is effectively limited by non-uniformities in the RF field. High efficiency transfers can require substantial RF power levels, and a tuned-RF circuit is needed to prevent large temperature excursions of the holding cryostat. In this paper, we compare the advantages and limitations of two different RF transfer methods to increase D polarization, Forbidden Adiabatic and Saturated Forbidden RF Transitions. The experience with the HD targets used during the recently completed E06-101 experiment in Hall-B of Jefferson Lab is discussed.
\end{abstract}

XVth International Workshop on Polarized Sources, Targets, and Polarimetry,

September 9-13, 2013

Charlottesville, Virginia, USA

\footnotetext{
* Speaker.

${ }^{\dagger}$ Present address: Le Moyne College, Syracuse, NY 13214 USA
} 


\section{Introduction}

Polarized HD in the frozen-spin mode has been used as a fixed target for nuclear physics measurements, especially with polarized photon beams, over the past decade [1]. The concept was initially developed at Syracuse [2] and subsequently engineered into a viable target for nuclear physics at the Laser-Electron-Gamma-Source (LEGS) facility at Brookhaven National Laboratory [3, 4]. There, beam-target double polarization experiments were carried out in the region of the $P_{33}(1232)$ $\Delta$ resonance [1]. At Jefferson Lab (JLab), a polarized HD target (known locally as HDice) has been successfully installed in the CLAS detector for the "complete" [5, 6] meson photo-production experiment E06-101 [7], which ran in 2011-2012 as the $g 14$ run and measured polarization observables from polarized neutrons in deuterium. Several targets were prepared and three of them were used in the experiment. During the course of the run, the directions of $\mathrm{H}$ and D were rotated and/or flipped to opposite directions, either together or individually, and the polarizations were transferred between $\mathrm{H}$ and $\mathrm{D}$. A pair of newly designed "Birdcage" coils was used to manipulate the $\mathrm{H}$ and $\mathrm{D}$ polarizations (designated below as $P_{H}$ and $P_{D}$, respectively), and to monitor the NMR signals.

A typical life cycle for an HDice target is sketched schematically in Figure 1. It includes the following three major steps:

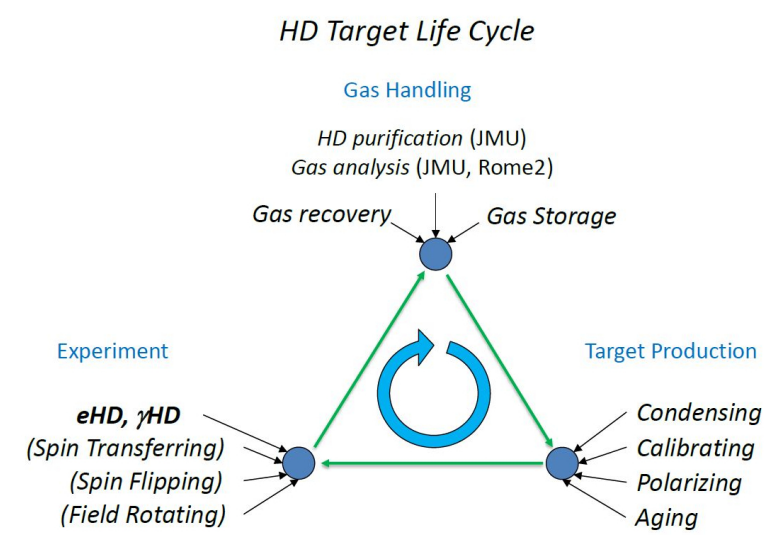

Figure 1: The life cycle of the ploarized HD target. Six cryostats with temperatures from $10 \mathrm{mK}$ to $18 \mathrm{~K}$ and magnetic fields up to $15 \mathrm{~T}$ are utilized during a target life time.

1.) HD GAS HANDLING - Raw HD, purchased from a commercial vendor, is purified to reduce the concentrations of $\mathrm{H}_{2}$ and $\mathrm{D}_{2}$ with a specially constructed distillation apparatus in order to be qualified as target grade gas. The impurity levels of $\mathrm{H}_{2}$ and $\mathrm{D}_{2}$ are measured with Gas Chromatography (GC) and Raman Spectrometry [8, 9].

2.) TARGET PRODUCTION_During which the HD gas is condensed into a target cell and the thermal equilibrium (TE) NMR signals of $\mathrm{H}$ and $\mathrm{D}$ are measured. The target is then transferred [10] into an Oxford Instruments Kelvinox-1000 dilution refrigerator and polarized at 10 milli-Kelvin $(\mathrm{mK})$ and 15 Tesla. As the $\mathrm{o}-\mathrm{H}_{2}$ and $\mathrm{p}-\mathrm{D}_{2}$ catalysts decay away, the target reaches the frozen-spin mode $[11,12]$.

3.) NUCLEAR PHYSICS EXPERIMENT-The frozen-spin target is transferred into an InBeam Cryostat (IBC) for photon or electron beam bombardment and data collection [13]. Depending upon the run requirement, the polarizations of $\mathrm{H}$ and $\mathrm{D}$ are manipulated together or separately, 
and/or transferred from one to the other. This is essential for the extraction of some polarization asymmetries and greatly aids the reduction of systematic uncertainties. Transferring spin from $\mathrm{H}$ to D significantly increases the figure of merit for polarized neutron experiments. Finally, the used target is evaporated after a post run calibration and the purities of the recovered HD are examined before reentering the HD stock.

\section{Spin Manipulation Methods}

The magnetic moments of $\mathrm{H}$ and $\mathrm{D}$ have the same sign and the spins of $\mathrm{H}$ and $\mathrm{D}$ are aligned with the magnetic field after the target is polarized with high field and low temperature. Figure 2 shows the thermal equilibrium polarizations of $\mathrm{H}$ and $\mathrm{D}$ at different polarizing conditions. Because the HDice targets are polarized at 15 Tesla and $10 \mathrm{mK}$, the $T E$ polarization for $\mathrm{H}$ is $91 \%$ and for $\mathrm{D}$ is $31 \%$. The heat generated from decay of $\mathrm{L}=1 \mathrm{H}_{2}$ and $\mathrm{D}_{2}$ impurities which are needed for the polarizing process, the Kapitza resistance between HD and aluminum cooling wires, and the low thermal conductivities of relevant materials along the heat path at milli-Kelvin temperature prevent the target from reaching the $T E$ polarizations. In practice, the $P_{H}$ and $P_{D}$ always freeze out at about $60 \%$ and $18 \%$, respectively, at least in the large-scale targets used in nuclear physics experiments.

\section{HD Equilibrium Polarization}

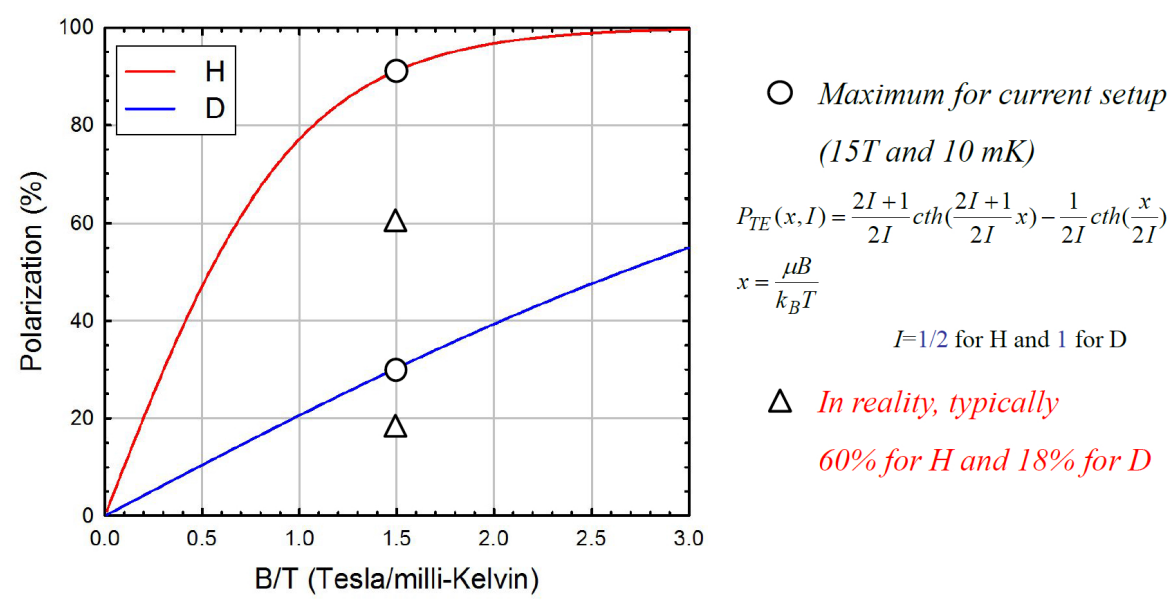

Figure 2: TE polarizations for $\mathrm{H}$ and $\mathrm{D}$ in different fields and temperatures. In reality, for various reasons, the target polarizations of $\mathrm{H}$ and $\mathrm{D}$ were always around $60 \%$ and $18 \%$, respectively.

$P_{H}$ and $P_{D}$ can be manipulated by rotating the target holding magnetic field, by flipping $P_{H}$ and/or $P_{D}$, and by applying RF to transfer spin between them. All of these methods have in fact been used on the same target during the $g 14$ run at Jefferson Lab. To move both $P_{H}$ and $P_{D}$ together to the opposite direction, the polarization rotation process, shown in Figure 3, has been used. Two specially arranged magnets, which generate orthogonal fields that overlap each other in the target region, are used to adiabatically rotate the spins. The holding field, $\mathbf{B}_{\|}$, is ramped to its opposite direction, while the transverse field, $\mathrm{B}_{\perp}$, is ramped up and down to zero synchronously, to ensure the magnetic field in target region, $\left|\left(B_{\|}^{2}+B_{\perp}^{2}\right)^{\frac{1}{2}}\right|$, always exceeds the minimum required value. $P_{H}$ 


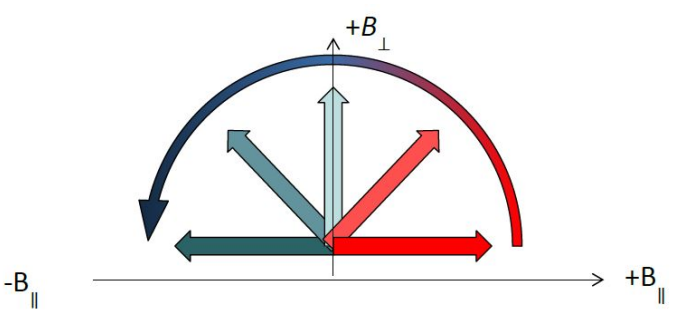

Figure 3: Polarization rotation process. Two specially arranged magnets, which generate orthogonal fields that overlap each other in the target region, are used to adiabatically rotate the spins. The spins of $\mathrm{H}$ and $\mathrm{D}$ will follow the direction of the total field.

and $P_{D}$ will adiabatically follow the total field, as long as the rate of direction change is small compared to the Larmor frequencies.

For separate flipping of $\mathrm{H}$ or D polarizations in the target, the allowed RF transitions are used. Either $P_{H}$ or $P_{D}$ can each be reversed in sign independently by applying RF power to adiabatically flip all spins of a species. Figure 4a illustrates the Zeeman levels of the HD system and the polarization reversal processes. The $\mathrm{m}_{H}$ substates (or the $\mathrm{m}_{D}$ substates) can be connected with $\mathrm{RF}$ at their Larmor frequencies, shown in blue for $\gamma_{H}$ (and in red for $\gamma_{D}$ ). $P_{H}$ and $P_{D}$ can be individually reversed by applying RF power to the HD system at $\gamma_{H}=42.5776 \mathrm{MHz} /$ Tesla or $\gamma_{D}=$ 6.5359MHz/Tesla, respectively, while adiabatically sweeping all HD through the Larmor condition once. Multiple partial reversals will reduce the population difference and eventually equalize the population on the related states. For erasing polarization, modulated RF power and/or field sweep can be used to speed up the process.
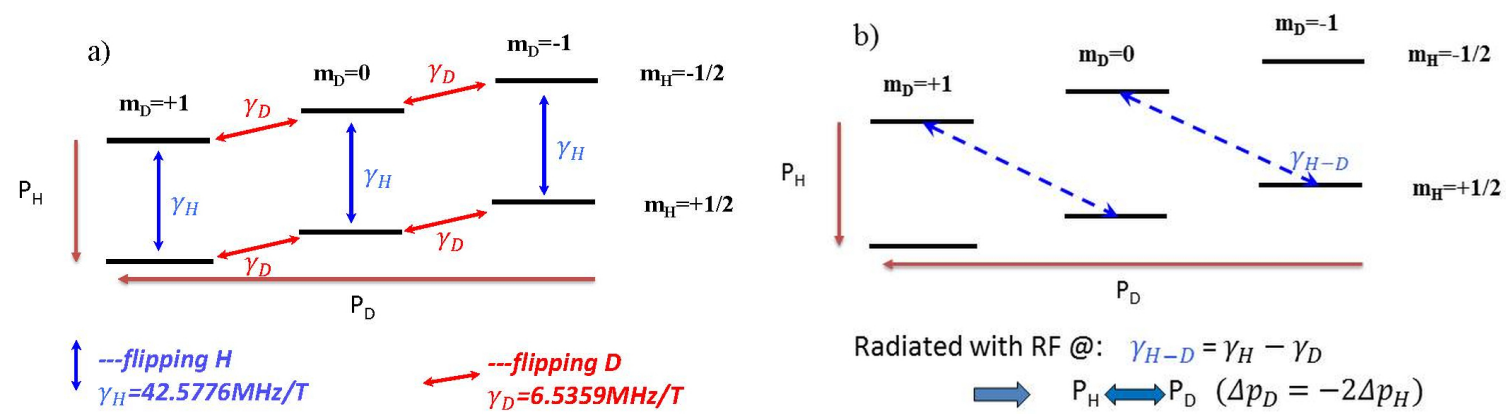

Figure 4: RF Transitions in HD system. a). Reverse or erase polarizations individually with allowed $R F$ transitions. b). Transfer polarizations between $\mathrm{H}$ and $\mathrm{D}$ with forbidden adiabatic fast passage (FAFP) or forbidden saturated fast passage (FSFP).

Spin flips that move polarization from $\mathrm{H}$ to $\mathrm{D}$ are forbidden, because two transitions must occur simultaneously. Within one molecule, the molecular potential has a repulsive core and the $\mathrm{H}$ and $\mathrm{D}$ nuclei are in a relative $\mathrm{S}$-state so that no net dipole-dipole interaction is possible. The most important remaining interaction is between adjacent molecules $[14,15]$, where an $\mathrm{H}$ can undergo a flip from $m_{H}=+\frac{1}{2}$ to $-\frac{1}{2}$, while a D makes a $1 \hbar$ transition between magnetic substates, as illustrated in Figure 4b. The final polarizations, ${ }^{f} P_{H}$ and ${ }^{f} P_{D}$, resulting from an RF transition driven at the difference of the Larmor frequencies of $\mathrm{H}$ and $\mathrm{D}\left(\gamma_{H-D}\right)$ are determined by equations (2.1) through (2.4). Here ${ }^{i} P_{H}$ and ${ }^{i} P_{D}$ are the initial vector polarizations, ${ }^{i} T_{D}$ is the initial deuteron tensor 
polarization (assumed to be determined by the equilibrium spin temperature), and the transition efficiency $(\varepsilon)$ is defined as the fraction of the population exchanged.

$$
\begin{gathered}
{ }^{f} P_{H}=\left(1-\frac{4}{3} \varepsilon\right){ }^{i} P_{H}+\varepsilon{ }^{i} P_{D}+\frac{1}{3} \varepsilon^{i} T_{D}{ }^{i} P_{H} \\
{ }^{f} P_{D}=\frac{2}{3} \varepsilon{ }^{i} P_{H}+\left(1-\frac{\varepsilon}{2}\right){ }^{i} P_{D}-\frac{1}{6} \varepsilon{ }^{i} T_{D}{ }^{i} P_{H} \\
{ }^{f} T_{D}=\left(1-\frac{3}{2} \varepsilon\right){ }^{i} T_{D}-\frac{3}{2} \varepsilon{ }^{i} P_{H}{ }^{i} P_{D} \\
{ }^{i} T_{D}=2-\left(4-3{ }^{i} P_{D}^{2}\right)^{\frac{l}{2}}
\end{gathered}
$$

In principle, the forbidden adiabatic fast passage (FAFP) of Figure $4 \mathrm{~b}$ can be $100 \%$ efficient. But this requires much more RF power applied to the HD system than the allowed RF transition. FAFP also requires a monotonic field sweep so all spins are flipped in a single pass. Those requirements are hard to satisfy without side effects. Higher than $50 \%$ efficiencies are not hard to achieve for a small sample in a test cryostat, where relatively large space, high cooling power and undistorted high power RF are available. But these are very hard to obtain for a large target inside an In-Beam Cryostat (IBC), where the geometry near the target is driven by the surrounding detectors, the materials used are limited by radiation length, and the cooling power is always limited and optimized for the "normal experimental running condition". For an HD target mounted inside an IBC, most of the applied RF power for inducing transitions is typically reflected back to the RF amplifier, while the associated Joule heating through the RF cables and Eddie current heating of the NMR coils will warm the IBC, which could then shorten the target relaxation time and result in a potential loss of polarization.

Alternatively, a forbidden saturated fast passage (FSFP) equalizes the populations in the connected levels of Figure 4b. Since this can be carried out with multiple applications of RF, it does not require a large efficiency per application. In practice, the RF is modulated and the magnetic field is swept repeatedly over the $\gamma_{H-D}$ line until no more net spin is transferred. This method greatly reduces the RF power requirement so that the in situ polarization transfer becomes practical and reliable during nuclear physics experiments. While the net efficiency of FSFP is only 50\%, it is a guaranteed $50 \%$.

\section{Experimental Setup}

The new IBC, a horizontal dilution refrigerator which can be operated stably in both horizontal and vertical positions and during the rotation, was constructed in 2011 for the E06-101[12] experiment in Hall B at Jefferson Lab. Since this device had to fit into the existing CLAS detector, several special geometry constraints had to be followed. In particular, the NMR coils were located outside the removable HD target cell and surrounded by an aluminum can which held both axial and transverse superconducting magnets. These coils needed to be designed to generate fairly uniform RF fields so the FSFP could be carried out throughout the entire target with minimum RF power. This requirement not only reduced the target heating, which could cause polarization loss, but also 


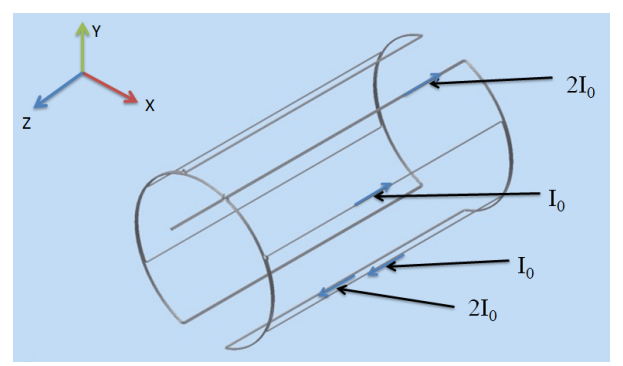

Figure 5: Birdcage coil designed for manipulating spins and monitoring NMR signals. On each side of the symmetric plane (Y-Z plane), a small signle-loop coil and a larger 2-loop coil are wound on a cylindrical surface. This arrangement doubles the current density near the two loop coil.

minimized the heating of the entire cryostat, which would result in operational instability. In order to match these requirements, a pair of Birdcage coils were designed and installed in the IBC for transferring $P_{H}$ to $P_{D}$, flipping or erasing target polarizations, and measuring NMR signals to track $P_{H}$ and $P_{D}$. Figure 5 shows a 3D CAD model of one coil. On each side of the symmetric plane (Y-Z plane), a small signle-loop coil and a larger 2-loop coil are wound on a cylindrical surface. This arrangement allows a doubling of the current density near the two loop coil. This 8-post coil, with varying current density along the different legs, generated a relatively uniform transverse RF field around the HD target, with about 10\% inhomogeneity. Figure 6 displays the results of the simulation of this Birdcage coil, carried out with CST Studio Suite 2013. [16]

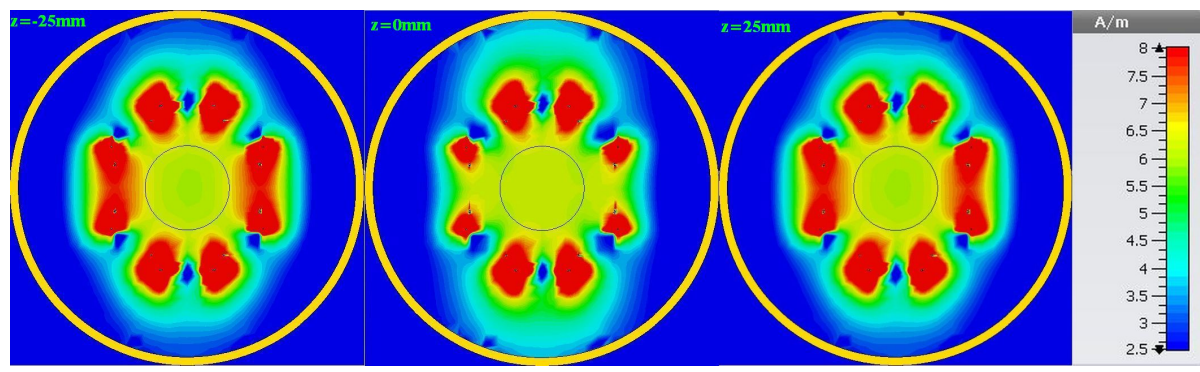

Figure 6: Transverse RF field simulation of the Birdcage coil, done with CST Studio Suite 2013. The RF field map at 3 locations, $-25 \mathrm{~mm}, 0 \mathrm{~mm}$ and $+25 \mathrm{~mm}$ (corresponding to upstream end, center and downstream end) is displayed with relative amplitude on the right. The black circles at the field map centers show the edge of solid HD.

The HDice target is a cylinder of solid HD, $15 \mathrm{~mm}$ in diameter and $50 \mathrm{~mm}$ long. The transverse RF field at 3 different locations, $-25 \mathrm{~mm}, 0 \mathrm{~mm}$ and $+25 \mathrm{~mm}$ (corresponding to upstream end, center and downstream end), are shown in Figure 6 with the linear relative amplitude scale on the right. The $15 \mathrm{~mm}$ diameter black circles on the field maps display the edge of solid HD and the black dots inside red, high field regions, are the posts of coils. Inside the target region, transverse field amplitudes are all around $6.0 \pm 0.1$. This improvement, combined with the in situ tuned RF power delivery circuit and SFP method, enabled us to make polarization manipulations as needed in the short Hall access times, which are mostly at the cost of precious beam time, during the $g 14$ run. 


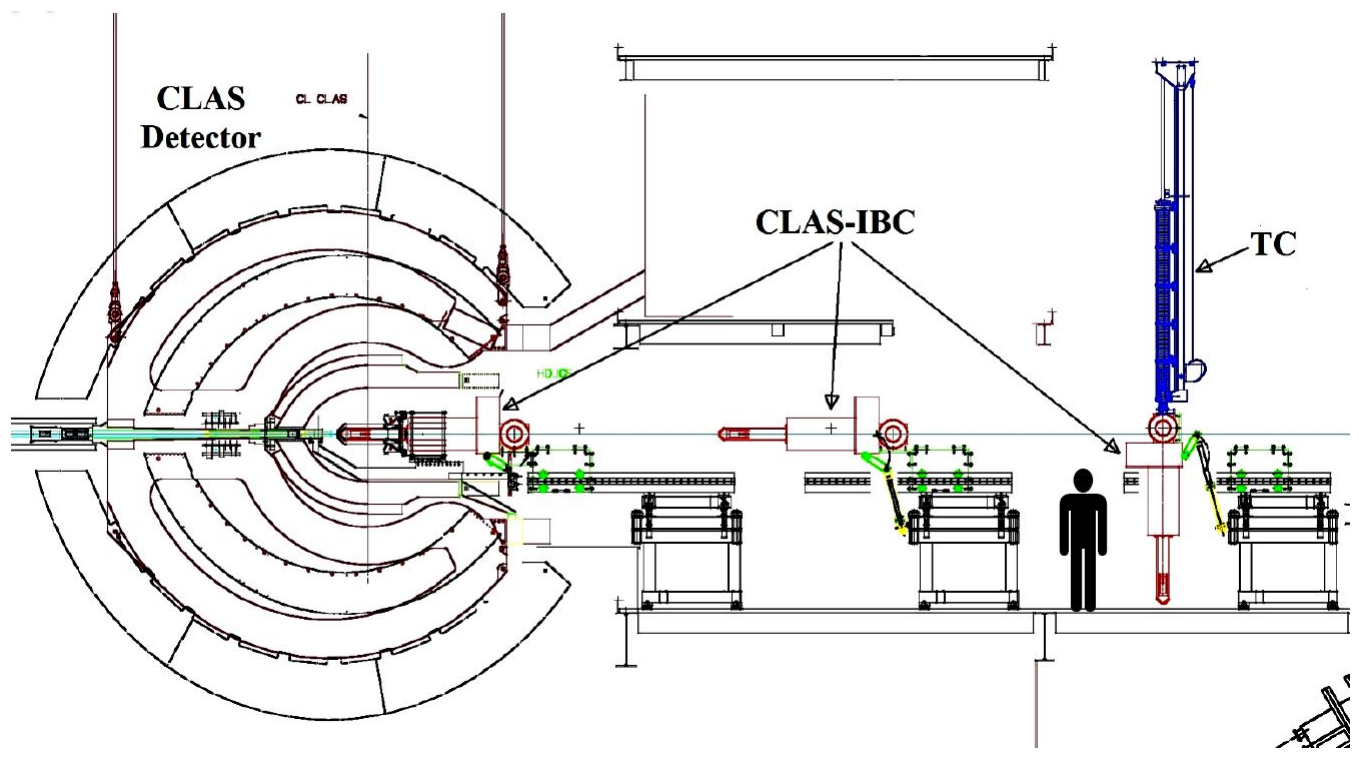

Figure 7: Setup for $g 14$ run with IBC at 3 different positions is shown here, from right to leftt, for 1) Loading target in vertical position, 2) Moving IBC with target inside, and 3) Taking nuclear physics data with CLAS, respectively.

\section{Target Performance During E06-101 Experiment}

The E06-101 Experiemnt, which used the HDice target, the CEBAF Large Acceptance Spectrometer (CLAS), and linearly and circularly polarized photon beams generated with the up to 6 GeV CEBAF polarized electron beams, was conducted at JLab during 2011-2012 $g 14$ run [7]. The Hall setup is shown in Figure 7, with a schematic figure standing by the IBC cart to show the size. CLAS, a $4 \pi$ detector surrounding the HDice IBC, is on the left. Three positions of the running IBC are displayed to show 1) Loading target in the vertical position, 2) Moving the IBC with the target inside, and 3) Taking nuclear physics data with CLAS. A detailed FSFP operation for target $19 \mathrm{~b}$ is

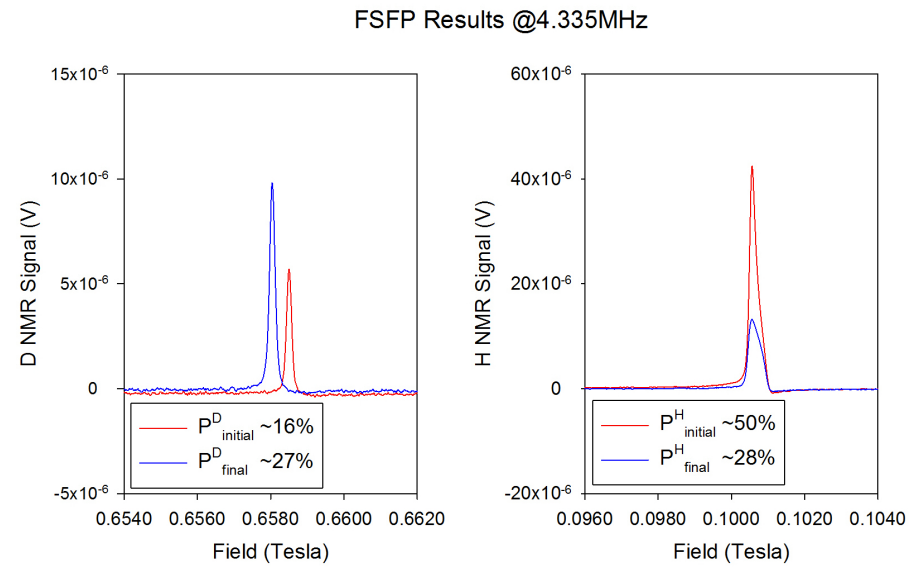

Figure 8: Details of the FSFP method to increase D polarzation during the $g 14$ run. The initial NMR signals are in red and final ones are in blue. $P_{D}$ increased from $16 \%$ to $27 \%$ after FSFP. 


\section{Target Polarizations during G-14 Run}

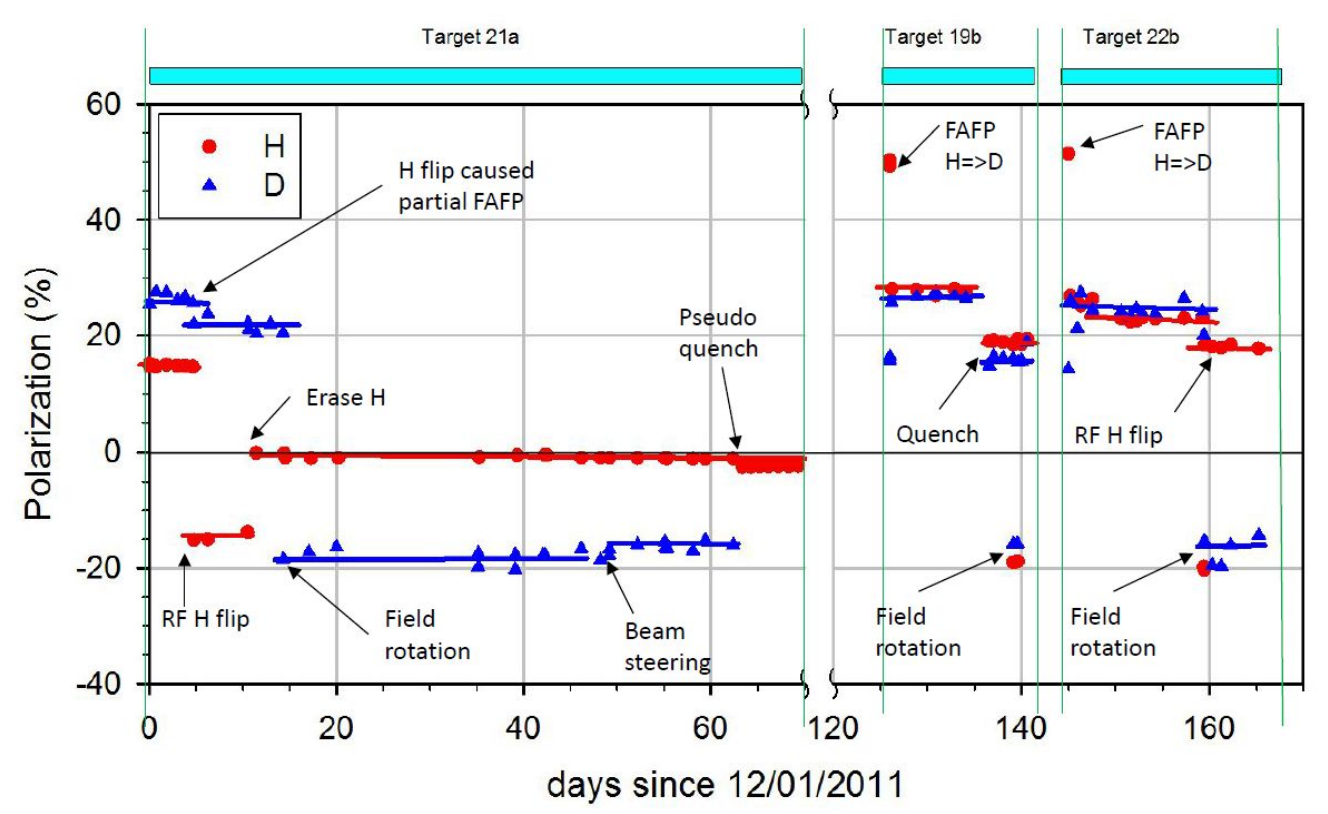

Figure 9: HDice target performance and polarization manuvers during $g 14$ run. The red circles and blue triangles are for $P_{H}$ to $P_{D}$, respectively. Positive polarization means the vector is aligned with the beam direction. The straight lines are used to guide the eyes. All spin maneuver methods for HDice target, mentioned in section 2 , were utilized during the run.

shown in Figure 8. The left panel is a plot of the D signal and the right panel is for $\mathrm{H}$. The initial NMR signals are in red and finals are in blue. $P_{D}$ increased from $16 \%$ to $27 \%$ after FSFP. Three HDice targets, denoted as 21a, 19b and 22b, were used successively during the g14 run. All targets were polarized inside a dilution refrigerator with $10 \mathrm{mK}$ and 15 Tesla, and transferred into the IBC within Hall-B. The performance of those targets are shown in Figure 9. The red circles and blue triangles are for $P_{H}$ to $P_{D}$, respectively. Positive polarization means the vector is aligned with the beam direction. The straight lines are used to guide the eyes. Target $21 \mathrm{a}$ came in after the FSFP had been performed in another cryostat, while the FSFP for targets $19 \mathrm{~b}$ and $22 \mathrm{~b}$ was performed after being loaded into the IBC. All spin maneuver methods for HDice targets, mentioned in section 2, were utilized during the run. Except for the loss due to operation error (partial FAFP), over steered beam, magnet quench, and deliberately erasing $P_{H}$, the polarizations of the targets stayed almost constant under normal IBC running conditions, $\mathrm{B}=0.9$ Tesla and $\mathrm{T}<100 \mathrm{mK}$.

After the $g 14$ run was finished in June 2012, data analysis has ramped up. Because of the simplicity of HDice targets, which contain 77\% HD, 17\% aluminum cooling wires (removable with empty cell subtraction), and 6\% pCTFE walls in the beam path (which can be removed with either vertex cuts or empty cell subtraction), data collected in the short run time still show the clear advantage of HD target with photon beam. Figure 10 shows the IBC structure near the target and the reconstructed vertex projections along the beam direction for both full target (in blue) and empty cell (in red). The contribution of HD lay between $-10.5 \mathrm{~cm}$ to $-5.5 \mathrm{~cm}$. The 3 peaks, from 


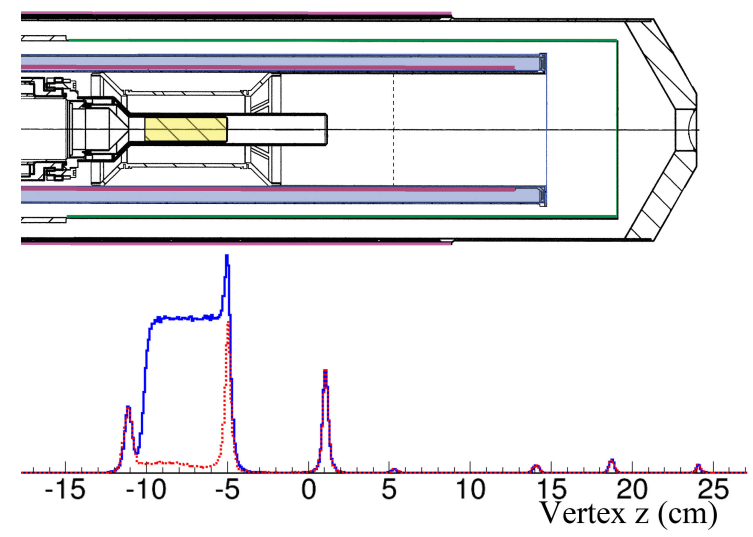

Figure 10: IBC structure near the target and the reconstructed vertex projections along the beam direction. The polarized solid HD is shown in yellow on upper penal, while the contribution from full target and empty cell are displayed in blue and red, respectively, on lower penal. The 3 peaks, from left to right, come from the target back-cap, front cap and a fixed pCTFE disc (for beam flux normalization), respectively.

left to right, come from the target back-cap, front cap and a fixed pCTFE disc (for beam flux normalization), respectively.A short list of ongoing analysis projects is shown here:

$$
\begin{aligned}
& \gamma \boldsymbol{n}(p) \longrightarrow K^{o} \Lambda(p) \\
& \gamma \boldsymbol{n}(p) \longrightarrow K^{-} \Sigma^{+}(p) \\
& \gamma \boldsymbol{n}(p) \longrightarrow \pi^{-} p(p) \\
& \gamma \boldsymbol{n}(p) \longrightarrow \pi^{+} \pi^{-} n(p) \Longleftrightarrow \pi^{+} \Delta^{-}(p), \pi^{-} \Delta^{+}(p), \rho n(p) \\
& \left.\gamma \boldsymbol{n}(p) \longrightarrow \pi^{+} \pi^{(} p\right) \Longleftrightarrow \eta n(p), \omega n(p) \\
& \gamma \boldsymbol{n}(p) \longrightarrow \pi^{o} \pi^{-} p(p)
\end{aligned}
$$

Figure 11 shows the preliminary results of E asymmetries from $\gamma \mathbf{n}(p) \longrightarrow \pi^{-} p(p)$ channel for 1 energy bin with only $10 \%$ of $g 14$ data processed and $27 \%$ D polarization[17]. Red and Green lines are predictions from MAID and SAID, respectively.

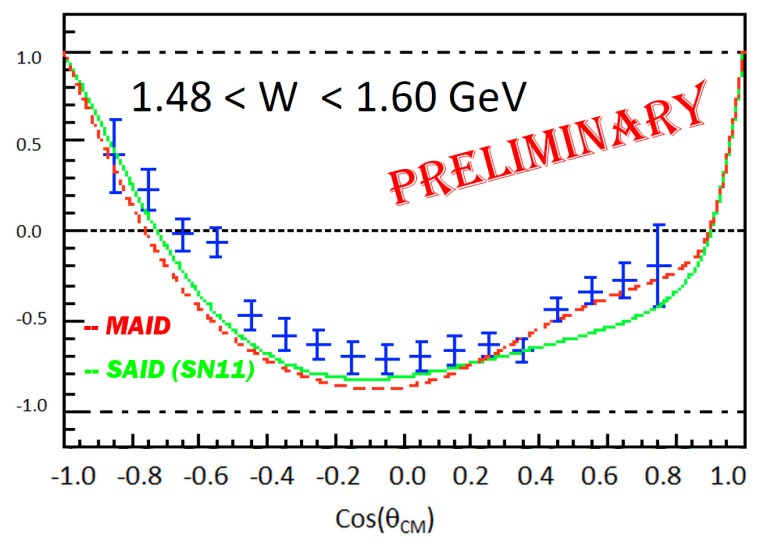

Figure 11: Preliminary results of $\mathrm{E}$ asymmetries from $\gamma \boldsymbol{n}(p) \longrightarrow \pi^{-} p(p)$ channel [17]. Red and Green lines are predictions from MAID and SAID, respectively. 


\section{Summary}

Methods of manipulating spins in HDice targets have been successfully implemented during the E06-101 run in CLAS. H and D Polarizations can be transferred back and forth. E06-101/g14 data from polarized $\mathrm{D}$, following FSFP, is now under analysis. These demonstrate a large potential for solid HD for photon experiments.

\section{Acknowledgments}

The authors are thankful to the JLab Hall-B technical crew for their technical support, and to the members of the CLAS collaboration for taking shifts to keep the target operational during the g14 Run. We are grateful to P. Collins, C. Hanretty, D. Ho, M. Khandaker, C. Nepali, P. Peng and $\mathrm{N}$. Walford for taking "around the clock" shifts during the target preparations and T. O'Connell for preparing target cells.

This work has been supported by the United States Department of Energy, Office of Nuclear Physics Division, under contract DEAC05-06OR23177 under which Jefferson Science Associates operates Jefferson Laboratory, the US National Science Foundation, and the Istituto Nazionale di Fisica Nucleare of Italy. The U.S. Government retains a non-exclusive, paid-up, irrevocable, world-wide license to publish or reproduce this manuscript for U.S. Government purposes.

\section{References}

[1] S. Hoblit et al., Phys Rev Lett 102 (2009) 172002.

[2] A. Honig, Phys Rev Lett 19 (1967) 1009

[3] X. Wei et al., NIM A526 (2004) 157.

[4] C. Thorn and A. Caracappa, AIP Conf. Proc. 980 (2008) 397.

[5] A. M. Sandorfi, J Phys Conf 424 (2013) 012001.

[6] A.M. Sandorfi, S. Hoblit, H. Kamano and T.-S.H. Lee, J Phys G38 (2011) 053001.

[7] F. Klein and A.M. Sandorfi et al., CLAS Collaboration Proposal, www.jlab.org/exp_prog/proposals/06/PR-06-101.pdf

[8] S. C. Whisnant et al. HD gas purification in proceedings of PSTP 2013, POS (PSTP 2013) 017

[9] A. D'Angelo et al., in proceedings of PST 2009, Ferrara, Italy, September 7-11, 2009, 123

[10] C. B. Bass et al., NIM A737 (2014) 107.

[11] A. Honig et al., NIM A356 (1995) 39.

[12] X. Wei et al., Physica B284-288 (2000) 2051.

[13] M. M. Lowry et al., Electrons on the HDice target in proceedings of PSTP 2013, POS (P STP 2013) 015

[14] A. Honig and H. Mano, Phys Rev B14 (1976) 1858.

[15] A. Abragam, Principles of Nuclear Magnetism, Chapter IX, (1983)

[16] https://www.cst.com/2013

[17] T. Kageya et al., in proceedings of $N \star 2013$, Peniscola, Spain, May 27-30, 2013 